\title{
Microbursts at Meter-Decameter Wavelengths
}

G. Thejappa* , N. Gopalswamy and M.R. Kundu Astronomy Program, University of Maryland, College Park, MD 20742

\section{Summary of Data Analysis}

A statistical analysis of Clark Lake microbursts was performed using a data base consisting of - 100 bursts. The results are: (1) Microburst sources are well correlated with coronal streamers and active regions. (2) The peak brightness temperature $\left(T_{b}\right)$ of the microbursts is in the range $6 \times 10^{5} \mathrm{~K}$ to $6 \times 10^{7} \mathrm{~K}$ (see Fig. 1; see also Kundu et al, 1986; White et al, 1986; Gopalswamy et al,1987) whereas it is quite high for normal type III bursts $\left(10^{7}<\mathrm{T}_{\mathrm{b}}<10^{12} \mathrm{~K}-\right.$ Dulk and Suzuki, 1980). (3) The source size varies over a wide range of $5.5-10.5$ arc min at $73.8 \mathrm{MHz}, 8.5-13.5$ arc min at $57.5 \mathrm{MHz}, 10.5-16.5$ arc mins at $50 \mathrm{MHz}$ and $12.5-22.5$ arc min at $38.5 \mathrm{MHz}$. (4) The drift rate lies mainly in the range 4.5 to $6.5 \mathrm{MHz} \mathrm{s}^{-1}$, with an average value of $\sim 5.5 \mathrm{MHz} \mathrm{s}^{-1}$.

\section{Interpretation and Discussion}

Most of the existing type III models consider the coherent emission of plasma waves by electron beams due to two-stream instability. Here we study both coherent and non-coherent emission of plasma waves by the electron beam in relation to microbursts. The intensity (I) of plasma waves emitted from a uniform plasma layer of thickness $L$ can be approximated as: $I=a / \mu$ where $a$ is the emissivity of the plasma waves and $\mu$ is the absorption coefficient which is the sum of collisional ( $\left.\mu_{\mathrm{coll}}\right)$, beam ( $\mu_{b}$ which is negative since it is growth) and Landau damping by ambient electrons $\mu_{L}$. The $\mu_{L}$ can be obtained by calculating the distance that a plasma wave can travel in smoothly varying corona before it is effectively Landau damped. Using the spontaneous emission due to the electron beam distribution, we obtain the total energy density of plasma waves from I as :

$$
\mathrm{W} / \mathrm{n}_{\mathrm{e}} \mathrm{T}_{\mathrm{e}} \approx 2 \times 10^{-2} \mathrm{n}_{\mathrm{b}} / \mathrm{n}_{\mathrm{e}} 1 / \mathrm{\gamma}
$$

where $\gamma$ is the effective damping due to all the above effects; $n_{e}, n_{b}$ are

* On leave from Indian Institute of Astrophysics, Bangalore, India. 521

E. R. Priest and V. Krishan (eds.), Basic Plasma Processes on the Sun, 521-522.

() 1990 IAU. Printed in the Netherlands. 
background and beam electron densities; $T_{e}$ is electron temperature. If the radiation is at fundamental plasma frequency due to Rayleigh scattering, the brightness temperature $T_{B}$ is obtained as:

$$
\mathrm{T}_{\mathrm{B}} \approx 6.0 \times 10^{13} \mathrm{n}_{\mathrm{b}} / \mathrm{n}_{\mathrm{e}} \frac{1}{\gamma}
$$

$\mathrm{T}_{\mathrm{B}}$ reaches maximum when growth balances collisional damping. At this threshold of $n_{b} / n_{e} \approx 9.2 \times 10^{-9}, T_{B} \approx 1.2 \times 10^{7} \mathrm{~K}$. $T_{B}$ is plotted as a function of density in Fig. 2. As $n_{b} / n_{e}$ approaches the threshold, $T_{B}$ rises sharply. It is also clear that the entire brightness spectrum of microbursts can be explained by the spontaneous emission mechanism. Thus the microbursts are due to spontaneously emitted electron beams in the range of densities $0.5 \times 10^{-8} \leq n_{b} / n_{e} \leqq 1 \times 10^{-8}$ with $V_{b} \approx \Delta V_{b} \approx 0.2 c$; the normal type III bursts are entirely in the coherent regime of Langmuir wave emission (see Thejappa et al 1989 for details).

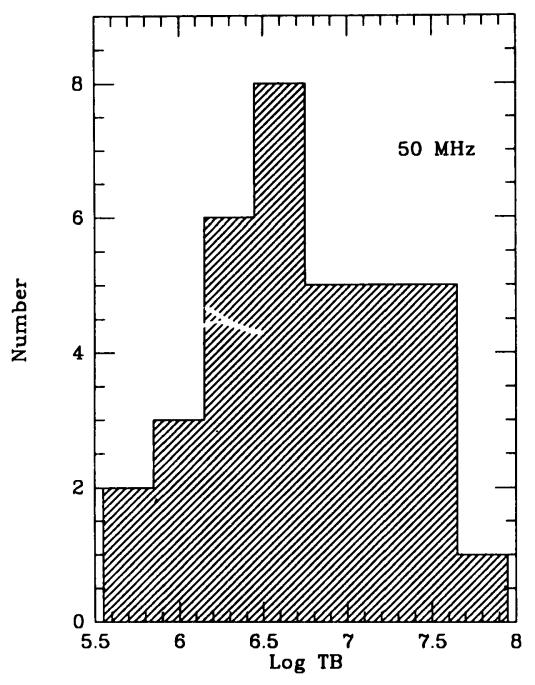

Fig. 1. Distribution of brightness temperature.

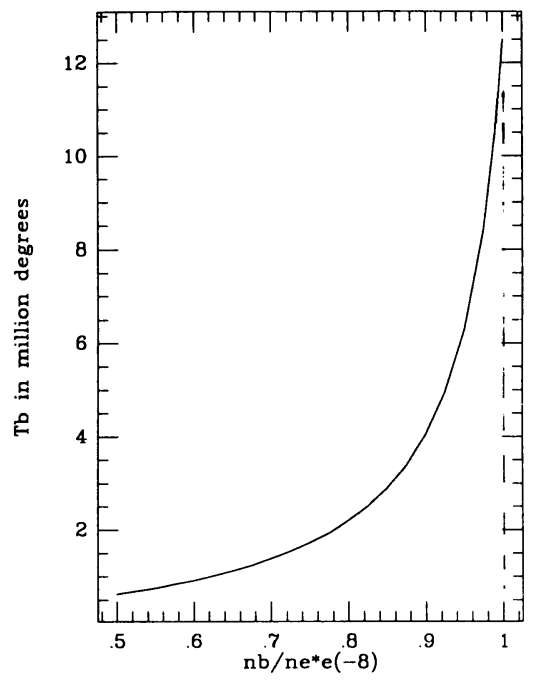

Fig. 2. Brightness temperature as a function of beam density. The region to the right of the dotted lines corresponds to coherent emission.

\section{References}

Dulk, G.A. and Suzuki, S.: 1980, Astron. Astrophys. 88, 203.

Gopalswamy, N., Kundu, M.R. and Szabo, A: 1987, Solar Phys. 108, 333. Kundu, M.R., Gergely, T.E., Szabo, A., Loiacono, R., and White, S.M.: 1986, Astrophpys. J. 308, 436.

Thejappa, G., Gopalswamy, N. and Kundu, M.R.: 1989, Solar Phys. (in press).

White, S.M., Kundu, M.R. and Szabo, A.: 1986, Solar Phys. 107, 135. 